\title{
The Spatial Relationship between Land Surface Temperature and Land Use/Cover Types in Sapporo City, Japan
}

\author{
Ruci Wang ${ }^{a}$, Yuji Murayama ${ }^{b}$ \\ ${ }^{a}$ Graduate School of Life and Environmental Science, University of Tsukuba, 1-1-1 Tennodai, Tsukuba City, \\ Ibaraki305-8572, Japan;warucy0720@yahoo.co.jp \\ ${ }^{b}$ Faculty of Life and Environmental Science, University of Tsukuba,1-1-1 Tennodai, Tsukuba City, \\ Ibaraki 305-8572, Japan; mura@geoenv.tsukuba.ac.jp
}

Key words: Geographical information science, Urban heat island, Land surface temperature, Land use/cover, Sapporo city

\begin{abstract}
:
Land surface temperature (LST) has received a significant attention for urban health and sustainable development. In recent years, with the rapid urbanization and industrialization, urban heat island phenomenon has prevailed in many cities in developed countries and caused excessive energy consumption, and climatic and environmental deterioration. It is expected that to optimize the composition of land use/cover distribution can reduce the urban heat island phenomenon. Therefore, monitoring the spatial changes and analysing its effects on land surface temperature are crucial for maintaining the healthy urban development in the near future.

Based on the above background, this study tried to geographically examine the relationship between the LST and land use/cover changes in Sapporo which is the fifth largest city and a gateway city on the northern island in Japan. We investigated the LST in each land use/cover category from 1985 to 2015 by using Landsat data. The results show that: (1) the land use/cover types and spatial distribution have a great influence on the formation of the LST; (2) the expansion of built-up area is the most significant factor affecting urban heat island during the past 30 years; and (3) the green space and water area help cool the urbanized area. By revealing a spatial relationship between the land use/cover distribution and the LST, this analysis could open an avenue to scientific research on future LST simulation and scenario based on land use/cover changes.
\end{abstract}

Our research goal does not only contribute to the urban health and sustainable development but also provide an important insight into the consequence of the strong city competence. 